TOPOLOGICAL ALGEBRAS, THEIR APPLICATIONS,

AND RELATED TOPICS

BANACH CENTER PUBLICATIONS, VOLUME 67

INSTITUTE OF MATHEMATICS

POLISH ACADEMY OF SCIENCES

WARSZAWA 2005

\title{
A CLASSIFICATION OF PROJECTORS
}

\author{
GUSTAVO CORACH \\ Departamento de Matemática, FI-UBA, and IAM-CONICET, Buenos Aires, Argentina \\ E-mail: gcorach@fi.uba.ar
}

ALEJANDRA MAESTRIPIERI

Instituto de Ciencias, UNGS, San Miguel, Argentina

E-mail: amaestri@ungs.edu.ar

DEMETRIO STOJANOFF

Departamento de Matemática, FCE-UNLP, La Plata, Argentina

E-mail: demetrio@mate.unlp.edu.ar

\begin{abstract}
A positive operator $A$ and a closed subspace $\mathcal{S}$ of a Hilbert space $\mathcal{H}$ are called compatible if there exists a projector $Q$ onto $\mathcal{S}$ such that $A Q=Q^{*} A$. Compatibility is shown to depend on the existence of certain decompositions of $\mathcal{H}$ and the ranges of $A$ and $A^{1 / 2}$. It also depends on a certain angle between $A(\mathcal{S})$ and the orthogonal of $\mathcal{S}$.
\end{abstract}

1. Introduction. Consider the set $\mathcal{Q}$ of all (bounded linear) projectors on a Hilbert space $\mathcal{H}$. Sometimes the elements of $\mathcal{Q}$ are named oblique projectors in order to emphasize that they are not necessarily orthogonal. Since the early years of matrix and operator theories, projectors have played a relevant role in many studies on spectral theory, approximation, optimization, orthogonal decompositions, least square methods, and so on. Very recently, several applications of oblique projectors to signal processing [10], [13], [36]; sampling [11], [57]; wavelets [3], [56]; information theory [57]; integral equations [51], [52]; statistics [54]; least square approximation [28], [29], [60] and parallel computing [17] have been found. For these multiple manifestations, many results on projectors are rediscovered once and again by different specialists. It seems that a short survey on several old and new results on oblique projectors may be helpful for the interested reader.

2000 Mathematics Subject Classification: 47A64, 47A07, 46C99.

Key words and phrases: oblique projectors, least square problems, scaled projectors, positive operators.

Research partially supported by CONICET (PIP 2083/02), Universidad de Buenos Aires (UBACYT X050), Universidad de La Plata 11 X350 and ANPCYT (PICT03-9521).

The paper is in final form and no version of it will be published elsewhere. 
For each closed subspace $\mathcal{S}$ of $\mathcal{H}$ let $\mathcal{Q}^{\mathcal{S}}$ denote the set of all projectors with range $\mathcal{S}$. For each (bounded linear semidefinite) positive operator $A$ on $\mathcal{H}$ consider the set $\mathcal{P}(A, \mathcal{S})=\left\{Q \in \mathcal{Q}^{\mathcal{S}}: A Q=Q^{*} A\right\}$, i.e., all $Q$ with range $\mathcal{S}$ which are Hermitian with respect to the sesquilinear form $\langle\xi, \eta\rangle_{A}=\langle A \xi, \eta\rangle$. Of course, $\mathcal{P}(A, \mathcal{S})$ can be empty (see examples below); we say that $A, \mathcal{S}$ are compatible if $\mathcal{P}(A, \mathcal{S})$ is not empty. This condition can be read in terms of different space decompositions, range inclusions and angles between certain closed subspaces of $\mathcal{H}$. It is known [19] that, if $A$ and $\mathcal{S}$ are compatible then a distinguished element $P_{A, \mathcal{S}}$ of $\mathcal{P}(A, \mathcal{S})$ exists which has optimal properties. We show explicit formulas for $P_{A, \mathcal{S}}$ which are computationally useful.

Many results on oblique projectors can be found in the papers by Afriat [1], Davis [22], Ljance [43], Mizel and Rao [45], Halmos [33], Greville [32], Gerisch [30], Pták [49]. Projectors which are Hermitian with respect to a positive matrix have been studied by Mitra and Rao [44] and Baksalary and Kala [9]. More recently, Hassi and Nordstrom [35] studied projectors which are Hermitian with respect to a self-adjoint operator but with emphasis on the case in which $\mathcal{P}(A, \mathcal{S})$ is a singleton. In [47], Pasternak-Winiarski studied the analyticity of the map $A \rightarrow P_{A, \mathcal{S}}$, where $A$ runs over the set of positive invertible operators. The map $(A, \mathcal{S}) \rightarrow P_{A, \mathcal{S}}$ is studied by Andruchow, Corach and Stojanoff [6], for positive invertible $A$. For general selfadjoint $A$, several results on $\mathcal{P}(A, \mathcal{S})$ can be found in [19] and the present paper can be seen as its continuation. Additional results by the authors are contained in [20] and [21]. The latter makes a link between oblique projectors and abstract splines in the sense of Atteia [8]. It is natural that this type of least square approximation results appears in this context, because $P_{A, \mathcal{S}}$ is a kind of orthogonal projector for an appropriate inner product. In particular, oblique projectors, mainly in the finite-dimensional setting, appear frequently under the form of "scaled projectors", i.e., projectors which are Hermitian with respect to a positive diagonal matrix. The reader is referred to the papers by Stewart [53], O'Leary [46], Hanke and Neumann [34], Gonzaga and Lara [31], Wei [60], Forsgren [28], Vavasis [14], among many others, for results on and applications of scaled projectors. A relationship between scaled and $A$-Hermitian projectors, also in the infinite-dimensional setting, can be found in [7].

The contents of the paper are the following. Section 2 begins with some preliminaries and a short survey of known results on $\mathcal{P}(A, \mathcal{S})$ and $P_{A, \mathcal{S}}$, taken from [19], [20] and [21]. Then, we prove several characterizations of compatibility in terms of decompositions of $\mathcal{H}$ and of the ranges of $A$ and $A^{1 / 2}$, of certain range inclusions and also of the angle between the closure of $A(\mathcal{S})$ with the orthogonal complement of $\mathcal{S}$. Most of these results are new and the proof of the remainder has been greatly simplified. We collect in Section 3 several formulas for $P_{A, \mathcal{S}}$ using results from Greville [32], Kerzman and Stein [38], [39], Ljance [43], Pták [49] and Buckholtz [16].

Acknowledgements. The first author thanks the Abdus Salam International Centre for Theoretical Physics for its kind hospitality.

2. Oblique projectors. In what follows $\mathcal{H}$ denotes a Hilbert space with inner product $\langle\rangle,, L(\mathcal{H})$ is the algebra of bounded linear operators on $\mathcal{H}, G L(\mathcal{H})$ denotes the group of invertible operators on $\mathcal{H}, L(\mathcal{H})^{+}$the cone of positive operators, $G L(\mathcal{H})^{+}=G L(\mathcal{H}) \cap$ 
$L(\mathcal{H})^{+}$and $\mathcal{Q}=\left\{Q \in L(\mathcal{H}): Q^{2}=Q\right\}$ the set of oblique projectors. For an operator $W$ its image is denoted by $R(W)$ and its nullspace by $N(W)$. Recall that if $\mathcal{H}, \mathcal{K}$ are two Hilbert spaces and $C \in L(\mathcal{H}, \mathcal{K})$ has closed range, then there exists a unique $C^{\dagger} \in L(\mathcal{K}, \mathcal{H})$ such that $C C^{\dagger} C=C, C^{\dagger} C C^{\dagger}=C^{\dagger}$ and $C C^{\dagger}, C^{\dagger} C$ are Hermitian; $C^{\dagger}$ is called the Moore-Penrose inverse of $C$ (see [23] and [12] for details).

The following result by R. G. Douglas will be frequently used in this paper. Given Hilbert spaces $\mathcal{H}, \mathcal{K}, \mathcal{G}$ and operators $A \in L(\mathcal{H}, \mathcal{G}), B \in L(\mathcal{K}, \mathcal{G})$ then the following conditions are equivalent:

i) the equation $A X=B$ has a solution in $L(\mathcal{K}, \mathcal{H})$;

ii) $R(B) \subseteq R(A)$;

iii) there exists $\lambda>0$ such that $B B^{*} \leq \lambda A A^{*}$.

In this case, there exists a unique $D \in L(\mathcal{K}, \mathcal{H})$ such that $A D=B$ and $R(D) \subseteq \overline{R\left(A^{*}\right)}$; moreover, $\|D\|^{2}=\inf \left\{\lambda>0: B B^{*} \leq \lambda A A^{*}\right\}$. We shall call $D$ the reduced solution of $A X=B$. The reader is referred to [26] and [27] for the proof of the Douglas theorem and related results. Let us remark that if $R(A)$ is closed then the reduced solution of $A X=B$ is $A^{\dagger} B$ : this follows quite easily from the properties of the Moore-Penrose pseudoinverse. For a fixed closed subspace $\mathcal{S}$ of $\mathcal{H}$, operators in $\mathcal{H}$ are represented as $2 \times 2$ matrices according to the decomposition $\mathcal{H}=\mathcal{S} \oplus \mathcal{S}^{\perp}$; more precisely, for each $B \in L(\mathcal{H})$ the identity

$$
B=P B P+P B(I-P)+(I-P) B P+(I-P) B(I-P)
$$

where $P$ is the orthogonal projector onto $\mathcal{S}$, can be matricially rephrased as $B=$ $\left(\begin{array}{ll}b_{11} & b_{12} \\ b_{21} & b_{22}\end{array}\right)$, where $b_{11}=\left.P B P\right|_{\mathcal{S}} \in L(\mathcal{S}), b_{12}=\left.P B(I-P)\right|_{\mathcal{S}^{\perp}} \in L\left(\mathcal{S}^{\perp}, \mathcal{S}\right), b_{21}=$ $\left.(I-P) B P\right|_{\mathcal{S}} \in L\left(\mathcal{S}, \mathcal{S}^{\perp}\right)$ and $b_{22}=\left.(I-P) B(I-P)\right|_{\mathcal{S}^{\perp}} \in L\left(\mathcal{S}^{\perp}\right)$. In particular, $P=\left(\begin{array}{ll}1 & 0 \\ 0 & 0\end{array}\right)$, any projector $Q$ onto $\mathcal{S}$ has the form $Q=\left(\begin{array}{ll}1 & e \\ 0 & 0\end{array}\right)$ for some $e \in L\left(\mathcal{S}^{\perp}, \mathcal{S}\right)$ and any $A \in L(\mathcal{H})^{+}$can be expressed as $A=\left(\begin{array}{cc}a & b \\ b^{*} & c\end{array}\right)$, where $a \in L(\mathcal{S})^{+}, b \in L\left(\mathcal{S}^{\perp}, \mathcal{S}\right)$, $c \in L\left(\mathcal{S}^{\perp}\right)^{+}$and $|\langle b \eta, \xi\rangle|^{2} \leq\langle a \xi, \xi\rangle\langle c \eta, \eta\rangle$ for every $\xi \in \mathcal{S}, \eta \in \mathcal{S}^{\perp}$ [50]. As a consequence (see [4]) it follows that the image of the positive square root of $a$ contains the image of $b: R\left(a^{1 / 2}\right) \supseteq R(b)$.

Given a closed subspace $\mathcal{S}$ let $\mathcal{Q}^{\mathcal{S}}$ be the subset of $\mathcal{Q}$ of all projectors with range (i.e. image) $\mathcal{S}$. Of course $\mathcal{Q}$ is the disjoint union of all $\mathcal{Q}^{\mathcal{S}}$. On the other hand, any positive (bounded linear) operator $A$ on $\mathcal{H}$ defines a (Hermitian semidefinite) positive sesquilinear form

$$
\langle,\rangle_{A}: \mathcal{H} \times \mathcal{H} \rightarrow \mathbb{C}, \quad\langle\xi, \eta\rangle_{A}=\langle A \xi, \eta\rangle, \quad \xi, \eta \in \mathcal{H}
$$

A (bounded linear) operator $T$ on $\mathcal{H}$ is called $A$-Hermitian if $\langle T \xi, \eta\rangle_{A}=\langle\xi, T \eta\rangle_{A}$ for all $\xi, \eta \in \mathcal{H}$, i.e. if $A T=T^{*} A$. We shall not study the existence of an $A$-adjoint of an operator (see [41] and [25] for this type of problems). However, the following result shows that this existence is not irrelevant, even in the case of projectors. 
Lemma 2.1. Given $Q \in \mathcal{Q}$ and $A \in L(\mathcal{H})^{+}$, there exists $W \in L(\mathcal{H})$ such that $A Q=W^{*} A$ (i.e. $Q$ admits an $A$-adjoint) if and only if

$$
R(A)=R(A) \cap N\left(Q^{*}\right) \oplus R(A) \cap R\left(Q^{*}\right) .
$$

Proof. If $\xi \in R(A)$ then $\xi=A \eta$, for some $\eta \in \mathcal{H}$. Since $Q^{*}$ decomposes $\mathcal{H}$ as the direct sum $R\left(Q^{*}\right) \oplus N\left(Q^{*}\right)$ there exists $w \in \mathcal{H}$ such that $\xi=A \eta=Q^{*} w+z$, where $z \in N\left(Q^{*}\right)$. But $Q^{*} \xi=Q^{*} A \eta=Q^{*} w \in R(A)$, because $R\left(Q^{*} A\right)=R(A W) \subseteq R(A)$. Then $Q^{*} \xi=Q^{*} w \in R(A) \cap R\left(Q^{*}\right)$. Also $z=A \eta-Q^{*} w \in R(A) \cap N\left(Q^{*}\right)$, because $Q^{*} w \in R(A)$. This proves decomposition (1).

If formula (1) holds, then $R\left(Q^{*} A\right)=Q^{*}\left(R(A) \cap R\left(Q^{*}\right)\right)=R(A) \cap R\left(Q^{*}\right)$, so that $R\left(Q^{*} A\right) \subseteq R(A)$. By the Douglas theorem there exists a solution $W$ of the equation $A X=Q^{*} A$.

Denote by $\mathcal{Q}_{A}$ the set of all $A$-Hermitian projectors on $\mathcal{H}$ and $\mathcal{P}(A, \mathcal{S})=\mathcal{Q}^{\mathcal{S}} \cap \mathcal{Q}_{A}$. In [19] it is remarked that every $Q \in \mathcal{Q}$ belongs to some $\mathcal{P}(A, \mathcal{S})$. Thus, $\mathcal{Q}=\cup \mathcal{P}(A, \mathcal{S})$ where $\mathcal{S}$ runs over the class of all closed subspaces on $\mathcal{H}$ and $A$ over a class of positive operators $A$. The sets $\mathcal{P}(A, \mathcal{S})$ are the object of our study.

We follow the terminology proposed by Ben-Israel and Greville [12]: the operator $Q: \xi \mapsto Q \xi$ which performs the projection is named projector, while $Q \xi$ is the projection of $\xi$ (under $Q$ ).

In what follows $\mathcal{S}$ denotes a closed subspace of $\mathcal{H}$ and $A$ denotes a positive operator on $\mathcal{H}$. Define

$$
\mathcal{S}^{\perp_{A}}:=\left\{\xi \in \mathcal{H}:\langle\xi, \eta\rangle_{A}=0 \forall \eta \in \mathcal{S}\right\}
$$

The identities $\mathcal{S}^{\perp_{A}}=A^{-1}\left(\mathcal{S}^{\perp}\right)=(A \mathcal{S})^{\perp}$ will be used without further mention. Observe that, if $A$ is invertible, then $\langle,\rangle_{A}$ is an inner product which is equivalent to $\langle$,$\rangle ; so$ that the subspace $\mathcal{S}$ admits a closed $A$-complement in $\left(\mathcal{H},\langle,\rangle_{A}\right)$, namely $\mathcal{S}^{\perp_{A}}$; thus, $\mathcal{H}=\mathcal{S} \oplus \mathcal{S}^{\perp_{A}}$. However, if $A$ is not invertible, such a complement may not exist. In fact, $\mathcal{S} \cap \mathcal{S}^{\perp_{A}}$ may be non-trivial and $\mathcal{S}+\mathcal{S}^{\perp_{A}}$ may be a proper non-closed subspace of $\mathcal{H}$ (see below).

The next theorem collects several well-known facts on projectors which are due to many mathematicians: Afriat [1], Greville [32], Pták [49], Chung [18], Buckholtz [16]. Indeed, the use of projectors is so extended that many results appear once again in papers in functional analysis, statistics, matrix analysis, signal processing, and so on.

THEOREM 2.2. If $\mathcal{S}$ and $\mathcal{N}$ are closed subspaces of a Hilbert space $\mathcal{H}$ then the following properties are equivalent:

1. $\mathcal{H}=\mathcal{S} \oplus \mathcal{N}$,

2. there exists $Q \in \mathcal{Q}$ such that $R(Q)=\mathcal{S}$, and $N(Q)=\mathcal{N}$,

3. $P_{\mathcal{S}}-P_{\mathcal{N}} \in G L(\mathcal{H})$,

4. $\left\|P_{\mathcal{S}}+P_{\mathcal{N}}-I\right\|<1$,

5. $\left.P_{\mathcal{S}^{\perp}}\right|_{\mathcal{N}}$ is injective and $P_{\mathcal{S}^{\perp}}(\mathcal{N})=\mathcal{S}^{\perp}$.

In that case $P_{\mathcal{S}} P_{\mathcal{N} \perp}$ has a closed range,

$$
\left\|P_{\mathcal{S}} P_{\mathcal{N}}\right\|=\left\|P_{\mathcal{N}} P_{\mathcal{S}}\right\|<1, \quad P_{\mathcal{S}}+P_{\mathcal{N}}-P_{\mathcal{N}} P_{\mathcal{S}} \in G L(\mathcal{H}), \quad P_{\mathcal{N}} P_{\mathcal{S}}-I \in G L(\mathcal{H})
$$


and the projector onto $\mathcal{S}$ with nullspace $\mathcal{N}$ is

$$
\begin{aligned}
P_{\mathcal{S} / / \mathcal{N}} & =\left(P_{\mathcal{S}} P_{\mathcal{N}^{\perp}}\right)^{\dagger}=\left(I-P_{\mathcal{N} \perp} P_{\mathcal{S}}\right)^{-1} P_{\mathcal{N}} \\
& =\left(I-P_{\mathcal{S}} P_{\mathcal{N}}\right)^{-1} P_{\mathcal{S}}\left(I-P_{\mathcal{S}} P_{\mathcal{N}}\right) \\
& =\left(I-P_{\mathcal{N}} P_{\mathcal{S}}\right)^{-1}\left(I-P_{\mathcal{N}}\right) \\
& =P_{\mathcal{S}}\left(P_{\mathcal{S}}+P_{\mathcal{N}}-P_{\mathcal{N}} P_{\mathcal{S}}\right)^{-1} .
\end{aligned}
$$

In particular, $\left\|P_{\mathcal{S} / / \mathcal{N}}\right\|=\left(I-\left\|P_{\mathcal{N}} P_{\mathcal{S}}\right\|^{2}\right)^{-1 / 2}$.

REMARK 2.3. There is a formula, due to Kerzman and Stein [38], [39], which expresses, given a projector $Q$, the unique orthogonal projector $P$ such that $R(P)=R(Q)$. Some of the expressions of $P_{\mathcal{S} / / \mathcal{N}}$ given above follow from Kerzman-Stein's formula.

Definition 2.4. Let $\mathcal{S}$ be a closed subspace of $\mathcal{H}$ and let $A \in L(\mathcal{H})^{+}$. We say that the pair $(A, \mathcal{S})$ is compatible if the set $\mathcal{P}(A, \mathcal{S})$ is not empty.

The following result, due to M. G. Krein [40], will be used, implicitly or explicitly, several times.

Lemma 2.5 (Krein). Let $Q$ be a projector with $R(Q)=\mathcal{S}$. Then $Q$ is A-Hermitian if and only if $N(Q) \subseteq A^{-1}\left(\mathcal{S}^{\perp}\right)$. In particular, $Q \in \mathcal{P}(A, \mathcal{S})$ if and only if $N(Q) \subseteq A^{-1}\left(\mathcal{S}^{\perp}\right)$, so that $(A, \mathcal{S})$ is compatible if and only if $\mathcal{H}=\mathcal{S}+A^{-1}\left(\mathcal{S}^{\perp}\right)$.

Proof. Suppose that $A Q=Q^{*} A$ and consider $\xi$ such that $\xi \in N(Q)$, then $\langle A \xi, Q \theta\rangle$ $=\left\langle Q^{*} A \xi, \theta\right\rangle=\langle A Q \xi, \theta\rangle=0$, for all $\theta \in \mathcal{H}$. Therefore $A \xi \in R(Q)^{\perp}$, or, equivalently, $\xi \in A^{-1}\left(R(Q)^{\perp}\right)$. Conversely, suppose that $N(Q) \subseteq A^{-1}\left(R(Q)^{\perp}\right)$ and consider $\xi, \eta \in \mathcal{H}$. Decompose $\xi=\nu+\rho$ and $\eta=\nu^{\prime}+\rho^{\prime}$, where $Q \rho=\rho, Q \rho^{\prime}=\rho^{\prime}$ and $Q \nu=Q \nu^{\prime}=0$. Then $\langle A Q \xi, \eta\rangle=\left\langle A Q \rho, \nu^{\prime}+\rho^{\prime}\right\rangle=\left\langle A \rho, \rho^{\prime}\right\rangle$ and $\left\langle Q^{*} A \xi, \eta\right\rangle=\left\langle A \rho, Q\left(\nu^{\prime}+\rho^{\prime}\right)\right\rangle=\left\langle A \rho, \rho^{\prime}\right\rangle$. Thus $A Q=Q^{*} A$.

Observe that two projectors $Q_{1}, Q_{2}$ on $\mathcal{H}$ such that $R\left(Q_{1}\right)=R\left(Q_{2}\right)$ and $N\left(Q_{1}\right) \subseteq$ $N\left(Q_{2}\right)$ are equal: every $\xi \in \mathcal{H}$ can be written as $\xi=\rho+\nu$ with $\rho \in R\left(Q_{1}\right), \nu \in N\left(Q_{1}\right)$; then $Q_{1} \xi=\rho$ and $Q_{2} \xi=\rho+Q_{2} \nu=\rho$ because $\nu \in N\left(Q_{1}\right) \subseteq N\left(Q_{2}\right)$. Using this remark, we prove the next result.

Corollary 2.6. The set $\mathcal{P}(A, \mathcal{S})$ is parametrized by the set of all direct complements of $\mathcal{S}$ contained in $A^{-1}\left(\mathcal{S}^{\perp}\right)$.

REMARK 2.7. If $\mathcal{S} \cap N(A)=\{0\}$ the pair $(A, \mathcal{S})$ is compatible if and only if $\overline{A(\mathcal{S})} \oplus \mathcal{S}^{\perp}$ is closed. Indeed if $\mathcal{M}, \mathcal{N}$ are closed subspaces, then $\mathcal{M}+\mathcal{N}$ is closed if and only if $\mathcal{M}^{\perp}+\mathcal{N}^{\perp}$ is closed (see theorem 4.8 of [37]); if $(A, \mathcal{S})$ is compatible then $\mathcal{S} \oplus A(\mathcal{S})^{\perp}=\mathcal{H}$, a fortiori $\mathcal{S}+A(\mathcal{S})^{\perp}$ is closed. Then $\mathcal{S}^{\perp}+\overline{A(\mathcal{S})}$ is closed. Moreover $\mathcal{S}^{\perp} \cap \overline{A(\mathcal{S})}=\left(\mathcal{S}+A(\mathcal{S})^{\perp}\right)^{\perp}=\{0\}$. Conversely, if $\mathcal{S}^{\perp} \oplus \overline{A(\mathcal{S})}$ is closed, then $\mathcal{S}^{\perp}+\overline{A(\mathcal{S})}=\overline{\mathcal{S}^{\perp}+\overline{A(\mathcal{S})}}=\left(\mathcal{S} \cap A(\mathcal{S})^{\perp}\right)^{\perp}=$ $(\mathcal{S} \cap N(A))^{\perp}=\mathcal{H}$. Again, if $\mathcal{H}=\mathcal{S}^{\perp}+\overline{A(\mathcal{S})}$ then $\mathcal{S}+A(\mathcal{S})^{\perp}$ is closed and $\left(\mathcal{S}+A(\mathcal{S})^{\perp}\right)^{\perp}=$ $\mathcal{S}^{\perp} \cap \overline{A(\mathcal{S})}=\{0\}$.

The following remarks may be helpful to understand the meaning of compatibility. With the $2 \times 2$ matrix representation mentioned above, if $Q$ is a projector onto $\mathcal{S}$ then 
$Q \in \mathcal{P}(A, \mathcal{S})$ if and only if

$$
\left(\begin{array}{cc}
a & b \\
b^{*} & c
\end{array}\right)\left(\begin{array}{ll}
1 & e \\
0 & 0
\end{array}\right)=\left(\begin{array}{cc}
1 & 0 \\
x^{*} & 0
\end{array}\right)\left(\begin{array}{cc}
a & b \\
b^{*} & c
\end{array}\right) .
$$

It is easy to see that the four equations reduce to a single one, namely, $a x=b$. By Douglas theorem, $a x=b$ has a solution if and only if $R(b) \subseteq R(a)$ and, in this case, there is a unique $d \in L\left(\mathcal{S}^{\perp}, \mathcal{S}\right)$ such that $a d=b$ and $R(d) \subseteq R(a)$.

As we saw, if $A=\left(\begin{array}{cc}a & b \\ b^{*} & c\end{array}\right) \in L(\mathcal{H})^{+}$then $R\left(a^{1 / 2}\right) \supseteq R(b)$. In general, $R(a) \subseteq$ $R\left(a^{1 / 2}\right) \subseteq \overline{R(a)}$. Then, there is no much place for $a, b$ to satisfy $R(b) \subseteq R\left(a^{1 / 2}\right)$ and not satisfy $R(b) \subseteq R(a)$. In fact, given $\mathcal{S}$, the set $\Upsilon_{\mathcal{S}}=\left\{B \in L(\mathcal{H})^{+}:(B, \mathcal{S})\right.$ is compatible $\}$ is everywhere dense in $L(\mathcal{H})^{+}$. Moreover, $G L(\mathcal{H})^{+}$is dense in $L(\mathcal{H})^{+}$and $G L(\mathcal{H})^{+} \subseteq \Upsilon_{\mathcal{S}}$. Indeed, from the comments above, if $A \in G L(\mathcal{H})^{+}$, then $a \in G L(\mathcal{S})^{+}$, so that the equation $a x=b$ has the unique solution $x=a^{-1} b$. Then $\mathcal{P}(A, \mathcal{S})=\left\{P_{A, \mathcal{S}}\right\}$, where $P_{A, \mathcal{S}}=\left(\begin{array}{cc}1 & a^{-1} b \\ 0 & 0\end{array}\right)$.

The following result, which contains another parametrization of $\mathcal{P}(A, \mathcal{S})$, in terms of the set of solutions in $L\left(\mathcal{S}^{\perp}, \mathcal{S}\right)$ of the equation $a x=b$, follows from the remarks above.

THEOREM 2.8. The pair $(A, \mathcal{S})$ is compatible if and only if $R(b) \subseteq R(a)$. In this case

$$
\begin{aligned}
\mathcal{P}(A, \mathcal{S}) & =\left\{P+P V(I-P): V \in L\left(\mathcal{S}^{\perp}, \mathcal{S}\right), P A P V=\left.P A\right|_{\mathcal{S}^{\perp}}\right\} \\
& =\left\{\left(\begin{array}{ll}
1 & x \\
0 & 0
\end{array}\right): a x=b\right\} .
\end{aligned}
$$

We summarize the conditions which are equivalent to compatibility in the next statement:

TheOREM 2.9. Given a closed subspace $\mathcal{S}$ of $\mathcal{H}$ and a positive operator $A$ on $\mathcal{H}$, the following conditions are equivalent:

1. $\mathcal{P}(A, \mathcal{S})$ is non-empty;

2. $\mathcal{S}+\mathcal{S}^{\perp_{A}}=\mathcal{H}$;

3. there exists a closed subspace $\mathcal{W} \subseteq \mathcal{S}^{\perp_{A}}$ such that $\mathcal{S} \oplus \mathcal{W}=\mathcal{H}$;

4. for the representation $A=\left(\begin{array}{cc}a & b \\ b^{*} & c\end{array}\right)$ of $A$ under the decomposition $\mathcal{H}=\mathcal{S} \oplus \mathcal{S}^{\perp}$, we have $R(b) \subseteq R(a)$.

Example 2.10. If $A \in L(\mathcal{H})^{+}$has a dense non-closed image in $\mathcal{H}$, then

$$
B=\left(\begin{array}{cc}
A & A^{1 / 2} \\
A^{1 / 2} & I
\end{array}\right)
$$

belongs to $L(\mathcal{H} \oplus \mathcal{H})^{+}$because $B=T T^{*}$ for $T: H \rightarrow \mathcal{H} \oplus \mathcal{H}$ defined by $T \xi=\left(A^{1 / 2} \xi, \xi\right)$. On the other hand, $R\left(A^{1 / 2}\right)$ properly contains $R(A)$, so that $B$ and $\mathcal{H} \oplus\{0\}$ are not compatible. In the same order of ideas, let $C=\left(\begin{array}{cc}A & 0 \\ 0 & 0\end{array}\right) \in L(\mathcal{H} \oplus \mathcal{H})^{+}$. Then $(C, \mathcal{H} \oplus\{0\})$ is a compatible pair and $R(C)=R(A)$ is non-closed. 
Suppose that $(A, \mathcal{S})$ is compatible. Define $P_{A, \mathcal{S}}$ the unique member of $\mathcal{P}(A, \mathcal{S})$ determined by the reduced solution $d$ of $a e=b: P_{A, \mathcal{S}}=\left(\begin{array}{ll}1 & d \\ 0 & 0\end{array}\right)$. Then $\mathcal{P}(A, \mathcal{S})$ is an affine manifold identified with $\left\{T \in L(\mathcal{H}):\left.T\right|_{\mathcal{S}}=0, T\left(\mathcal{S}^{\perp}\right) \subseteq \mathcal{N}\right\}$. In particular, $\mathcal{P}(A, \mathcal{S})$ has a unique element if and only if $\mathcal{N}=\{0\}$. If $\mathcal{N} \neq\{0\}$, then $\left\|P_{A, \mathcal{S}}\right\| \leq\|Q\|$ for all $Q \in \mathcal{P}(A, \mathcal{S})$. For a proof of these facts, see [19].

Theorem 2.11. Let $A$ and $\mathcal{S}$ be compatible. Denote by $\mathcal{N}=(A \mathcal{S})^{\perp} \cap \mathcal{S}=N(A) \cap \mathcal{S}$. Then $N\left(P_{A, \mathcal{S}}\right)=(A \mathcal{S})^{\perp} \ominus \mathcal{N}$.

Proof. Both projectors have the same image, namely $\mathcal{S}$. It suffices to show that $N\left(P_{A, \mathcal{S}}\right)$ $\subseteq(A \mathcal{S})^{\perp} \ominus \mathcal{N}$. Recall that $P_{A, \mathcal{S}}=\left(\begin{array}{ll}1 & d \\ 0 & 0\end{array}\right)$ where $A=\left(\begin{array}{cc}a & b \\ b^{*} & c\end{array}\right)$ and $d$ is the reduced solution of $a x=b$, i.e., $a d=b$ and $R(d) \subseteq \overline{R(a)}$. If $\xi=\sigma+\sigma^{\perp} \in N\left(P_{A, \mathcal{S}}\right)$ then $\sigma+d \sigma^{\perp}=0$ and $\xi=-d \sigma^{\perp}+\sigma^{\perp}$. We must prove $-d s^{\perp}+s^{\perp} \in \mathcal{W}=(A \mathcal{S})^{\perp} \ominus \mathcal{N}$. First, let us show $-d \sigma^{\perp}+\sigma^{\perp} \in(A \mathcal{S})^{\perp}$, or, equivalently, that $A\left(-d \sigma^{\perp}+\sigma^{\perp}\right) \in \mathcal{S}^{\perp}$; but $\left(-d \sigma^{\perp}+\sigma^{\perp}\right)=\left(\begin{array}{cc}a & b \\ b^{*} & c\end{array}\right)\left(\begin{array}{c}-d \sigma^{\perp} \\ \sigma^{\perp}\end{array}\right)=\left(\begin{array}{c}0 \\ -b^{*} d \sigma^{\perp}+c \sigma^{\perp}\end{array}\right) \in \mathcal{S}^{\perp}$. Next, we must show that $-d \sigma^{\perp}+\sigma^{\perp} \in(\mathcal{S} \cap N(A))^{\perp}$. By the definition of $d,-d \sigma^{\perp}=\lim a \sigma_{n}$ for some sequence $\left\{\sigma_{n}\right\}$ in $\mathcal{S}$. Given $\sigma \in \mathcal{S} \cap N(A), a \sigma=A \sigma=0$, so that $\left\langle-d \sigma^{\perp}+\sigma^{\perp}, \sigma\right\rangle=$ $\left\langle-d \sigma^{\perp}, \sigma\right\rangle=\lim \left\langle a \sigma_{n}, \sigma\right\rangle=\lim \left\langle\sigma_{n}, a \sigma\right\rangle=0$. This finishes the proof.

REMARK 2.12. Under additional hypotheses on $A$, other characterizations of compatibility and formulas for $P_{A, \mathcal{S}}$ can be used. We mention a sample of these, taken from [19] and $[20]$ :

1. If $R(P A P)$ is closed (or, equivalently, if $R\left(P A^{1 / 2}\right)$ or $A^{1 / 2}(\mathcal{S})$ are closed), then $(A, \mathcal{S})$ is compatible. Indeed, if $A=\left(\begin{array}{cc}a & b \\ b^{*} & c\end{array}\right)$, the positivity of $A$ implies that $R(b) \subseteq R\left(a^{1 / 2}\right)$ (see, e.g., [4]). If $R(P A P)=R(a)$ is closed, then $R(b) \subseteq R\left(a^{1 / 2}\right)=$ $R(a)$ so that $(A, \mathcal{S})$ is compatible, by Theorem 2.8 . In this case,

$$
P_{A, \mathcal{S}}=\left(\begin{array}{cc}
1 & a^{\dagger} b \\
0 & 0
\end{array}\right),
$$

since $a=P A P$ has closed range, and $a^{\dagger} b$ is the reduced solution of $a x=b$. In particular, if $\mathcal{N}=N(a)=N(A) \cap \mathcal{S}=\{0\}$ (i. e. $R(a)=\mathcal{S}$ ), one gets

$$
P_{A, \mathcal{S}}=(P A P)^{\dagger} P A \text {. }
$$

Otherwise, $P_{A, \mathcal{S}}=P_{\mathcal{N}}+(P A P)^{\dagger} P A$.

2. If $A$ has closed range then the following conditions are equivalent:

(a) The pair $(A, \mathcal{S})$ is compatible.

(b) $R(P A P)$ is closed.

(c) $R(A P)$ is closed.

(d) $\mathcal{S}^{\perp}+R(A)$ is closed.

3. If $P, Q$ are orthogonal projectors with $R(P)=\mathcal{S}$, then $(Q, \mathcal{S})$ is compatible if and only if $R(Q P)$ is closed. Moreover, if $(Q, \mathcal{S})$ is compatible, then $\mathcal{H}=\mathcal{S}+Q^{-1}\left(\mathcal{S}^{\perp}\right)=$ 
$\mathcal{S}+\left(R(Q) \cap \mathcal{S}^{\perp}\right)+N(Q)$ and, if $\mathcal{N}=N(Q) \cap \mathcal{S}$ and $\mathcal{M}=\mathcal{S} \ominus \mathcal{N}$, then

(a) $P_{Q, \mathcal{S}}=P_{\mathcal{N}}+P_{Q}, \mathcal{M}$.

(b) $\mathcal{M} \oplus\left(N(Q) \perp\left(R(Q) \cap \mathcal{S}^{\perp}\right)\right)=\mathcal{H}$, and $P_{Q}, \mathcal{M}$ is the projector onto $\mathcal{M}$ given by this decomposition.

(c) In the particular case that $N(Q) \cap \mathcal{S}=\{0\}=R(Q) \cap \mathcal{S}^{\perp}$, then

$$
\mathcal{S} \oplus N(Q)=\mathcal{H}
$$

and $P_{Q, \mathcal{S}}$ is the projector given by this decomposition, i.e., $N\left(P_{Q, \mathcal{S}}\right)=N(Q)$.

(d) $\left\|P_{Q, \mathcal{S}}\right\|=\left\|P_{Q}, \mathcal{M}\right\|=\left(1-\left\|(I-Q) P_{\mathcal{M}}\right\|^{2}\right)^{-1 / 2}$.

REMARK 2.13. Consider the following conditions:

1. The pair $(A, \mathcal{S})$ is compatible;

2. $A(\mathcal{S})$ is closed in $R(A)$;

3. $A^{-1}(\overline{A(\mathcal{S})})=\mathcal{S}+N(A)$;

4. $A^{1 / 2}(\mathcal{S})$ is closed in $R\left(A^{1 / 2}\right)$;

5. $\mathcal{S}+N(A)$ is closed;

6. $P_{\overline{R(A)}}(\mathcal{S})$ is closed, where $P_{\overline{R(A)}}$ is the orthogonal projector onto $\overline{R(A)}$.

A precise description of the relationships among them is provided by the implications: $1 \rightarrow 2 \leftrightarrow 3 \rightarrow 4 \rightarrow 5 \leftrightarrow 6$. Moreover, $(A, \mathcal{S})$ is compatible if and only if $P_{\overline{R(A)}}(\mathcal{S})$ is closed and $\left(A, P_{\overline{R(A)}}(\mathcal{S})\right)$ is compatible.

The next result is a characterization of compatibility in terms of orthogonal decompositions of $R(A)$ and $R\left(A^{1 / 2}\right)$.

Proposition 2.14. Given $A \in L(\mathcal{H})^{+}$, the following conditions are equivalent:

1. The pair $(A, \mathcal{S})$ is compatible.

2. $R(A)=A(\mathcal{S}) \oplus \mathcal{S}^{\perp} \cap R(A)$.

3. $R\left(A^{1 / 2}\right)=A^{1 / 2}(\mathcal{S}) \oplus A^{1 / 2}(\mathcal{S})^{\perp} \cap R\left(A^{1 / 2}\right)$.

4. If $\mathcal{M}=\overline{A^{1 / 2}(\mathcal{S})}$, then $R\left(P_{\mathcal{M}} A^{1 / 2}\right) \subseteq R\left(A^{1 / 2} P\right)$.

Proof. $1 \leftrightarrow 2$ : If $(A, \mathcal{S})$ is compatible then $\mathcal{H}=\mathcal{S}+A^{-1}\left(\mathcal{S}^{\perp}\right)$ so that

$$
R(A)=A(\mathcal{S})+A\left(A^{-1}\left(\mathcal{S}^{\perp}\right)\right)=A(\mathcal{S})+\mathcal{S}^{\perp} \cap R(A) ;
$$

conversely, if $R(A)=A(\mathcal{S}) \oplus \mathcal{S}^{\perp} \cap R(A)$, then $\mathcal{H}=A^{-1}(R(A))=A^{-1}(A(\mathcal{S}))+A^{-1}\left(\mathcal{S}^{\perp} \cap\right.$ $R(A))$. But $A^{-1}\left(\mathcal{S}^{\perp} \cap R(A)\right)=A^{-1}\left(\mathcal{S}^{\perp}\right)$ and $A^{-1}(A(\mathcal{S}))=S+N(A)$, so that $\mathcal{H}=$ $S+N(A)+A^{-1}\left(\mathcal{S}^{\perp}\right)=S+A^{-1}\left(\mathcal{S}^{\perp}\right)$, because $N(A) \subseteq A^{-1}\left(\mathcal{S}^{\perp}\right)$.

$1 \leftrightarrow 3$ : similar to $(1) \leftrightarrow(2)$.

$3 \leftrightarrow 4$ : If $y \in R\left(A^{1 / 2}\right)$ then $y=y_{1}+y_{2}$ for unique $y_{1} \in A^{1 / 2}(\mathcal{S})$ and $y_{2} \in A^{1 / 2}(\mathcal{S})^{\perp}$; but, then, $P_{\mathcal{M}}(y)=y_{1} \in A^{1 / 2}(\mathcal{S})=R\left(A^{1 / 2} P\right)$. The converse is similar.

As a consequence of Proposition 2.14, it is easy to see that $(A, \mathcal{S})$ is compatible if and only if $A^{1 / 2}(\mathcal{S})$ is closed in $R\left(A^{1 / 2}\right)$ and

$$
R\left(A^{1 / 2}\right)=\overline{A^{1 / 2}(\mathcal{S})} \cap R\left(A^{1 / 2}\right) \oplus A^{1 / 2}(\mathcal{S})^{\perp} \cap R\left(A^{1 / 2}\right) .
$$

More generally, given a closed subspace $\mathcal{S}$ of $\mathcal{H}$ and $\mathcal{W}=A^{-1 / 2}\left(\overline{A^{1 / 2}(\mathcal{S})}\right)$, then $(A, \mathcal{W})$ is compatible if and only if $R\left(A^{1 / 2}\right)=\overline{A^{1 / 2}(\mathcal{S})} \cap R\left(A^{1 / 2}\right) \oplus A^{1 / 2}(\mathcal{S})^{\perp} \cap R\left(A^{1 / 2}\right)$ : in fact, if $(A, \mathcal{W})$ is compatible then, by Proposition $2.14, R\left(A^{1 / 2}\right)=A^{1 / 2}(\mathcal{W})+A^{1 / 2}(\mathcal{W})^{\perp} \cap$ 
$R\left(A^{1 / 2}\right)$. On one hand, $A^{1 / 2}(\mathcal{W})=\overline{A^{1 / 2}(S)} \cap R\left(A^{1 / 2}\right)$; on the other hand, since $A^{1 / 2}(\mathcal{S}) \subseteq$ $A^{1 / 2}(\mathcal{W}) \subseteq \overline{A^{1 / 2}(\mathcal{S})}$, we get $A^{1 / 2}(\mathcal{S})^{\perp}=A^{1 / 2}(\mathcal{W})^{\perp}$. Thus,

$$
R\left(A^{1 / 2}\right)=\overline{A^{1 / 2}(\mathcal{S})} \cap R\left(A^{1 / 2}\right)+A^{1 / 2}(\mathcal{S})^{\perp} \cap R\left(A^{1 / 2}\right),
$$

and, of course, the sum is direct. The converse is similar.

A notion which is naturally related to oblique projectors is that of angle between subspaces. We consider here two non-equivalent definitions of angles and we show a characterization of the compatibility of $(A, \mathcal{S})$ in terms of these angles. For excellent treatments on angles in Hilbert spaces the reader is referred to the survey by Deutsch [24] or the book by A. Ben-Israel and T. N. E. Greville [12]

Given two subspaces $\mathcal{S}, \mathcal{T}$, the cosine of the Friedrichs angle between them is defined by

$$
c(\mathcal{S}, \mathcal{T})=\sup \left\{|\langle\xi, \eta\rangle|: \xi \in \mathcal{S} \cap(\mathcal{S} \cap \mathcal{T})^{\perp}, \quad\|\xi\|<1, \eta \in \mathcal{T} \cap(\mathcal{S} \cap \mathcal{T})^{\perp}, \quad\|\eta\|<1\right\} .
$$

It is well known (see Theorem 13 of [24]) that the following conditions are equivalent:

1. $c(\mathcal{S}, \mathcal{T})<1$

2. $\mathcal{S}+\mathcal{T}$ is closed;

3. $\mathcal{S}^{\perp}+\mathcal{T}^{\perp}$ is closed;

4. $c\left(\mathcal{S}^{\perp}, \mathcal{T}^{\perp}\right)<1$.

The formulas $\left\|P_{\mathcal{S}} P_{\mathcal{T}}\right\|=c(\mathcal{S}, \mathcal{T})[24]$ and $\left\|P_{\mathcal{S} / / \mathcal{T}}\right\|=\left(1-c(\mathcal{T}, \mathcal{S})^{2}\right)^{-1 / 2}[49]$ relate this notion with oblique projectors.

The minimal angle between $\mathcal{S}$ and $\mathcal{T}$ is the angle whose cosine is defined by

$$
c_{o}(\mathcal{S}, \mathcal{T})=\sup \{|\langle\xi, \eta\rangle|: \xi \in \mathcal{S},\|\xi\|<1, \quad \eta \in \mathcal{T},\|\eta\|<1\} .
$$

Observe that $c(\mathcal{S}, \mathcal{T}) \leq c_{0}(\mathcal{S}, \mathcal{T})$ and $c(\mathcal{S}, \mathcal{T})=c_{0}(\mathcal{S}, \mathcal{T})$ when $\mathcal{S} \cap \mathcal{T}=\{0\}$.

TheOrem 2.15. Consider $A \in L(\mathcal{H})^{+}$. Then $(A, \mathcal{S})$ is compatible if and only if $c_{0}\left(\mathcal{S}^{\perp}, \overline{A(\mathcal{S})}\right)<1$.

Proof. If $(A, \mathcal{S})$ is compatible then $\mathcal{H}=\mathcal{S}+A^{-1}\left(\mathcal{S}^{\perp}\right)$, so that $\mathcal{S}+A^{-1}\left(\mathcal{S}^{\perp}\right)$ is closed. By the remarks above and the identity $A^{-1}\left(\mathcal{S}^{\perp}\right)=(A \mathcal{S})^{\perp}$, we get $c\left(\mathcal{S}, A^{-1}\left(\mathcal{S}^{\perp}\right)\right)<1$ or equivalently, $c\left(\mathcal{S}^{\perp}, \overline{A(\mathcal{S})}\right)<1$. But $\mathcal{S}^{\perp} \cap \overline{A(\mathcal{S})}=\left(\mathcal{S}+A^{-1}\left(\mathcal{S}^{\perp}\right)\right)^{\perp}=\mathcal{H}^{\perp}=\{0\}$. Therefore, $c_{0}\left(\mathcal{S}^{\perp}, \overline{A(\mathcal{S})}\right)=c\left(\mathcal{S}^{\perp}, \overline{A(\mathcal{S})}\right)<1$.

Conversely, if $c_{0}\left(\mathcal{S}^{\perp}, \overline{A(\mathcal{S})}\right)<1$ then $\mathcal{S}^{\perp} \cap \overline{A(\mathcal{S})}=\{0\}$ and $\mathcal{S}^{\perp}+\overline{A(\mathcal{S})}$ is closed; therefore, $\mathcal{S}+A(\mathcal{S})^{\perp}$ is closed; also $\left(\mathcal{S}+A(\mathcal{S})^{\perp}\right)^{\perp}=\mathcal{S}^{\perp} \cap \overline{A(\mathcal{S})}=\{0\}$. Then $\mathcal{S}+A(\mathcal{S})^{\perp}=$ $\mathcal{H}$ and $(A, \mathcal{S})$ is compatible.

REMARK 2.16.

1. If $A$ has closed range then, by Remark 2.12 , the pair $(A, \mathcal{S})$ is compatible if and only if $R(A P)$ is closed. Note that this is equivalent to the angle condition $c(N(A), \mathcal{S})$ $<1$.

2. If $P, Q$ are orthogonal projectors with $R(P)=\mathcal{S}$, define $\mathcal{N}=N(Q) \cap \mathcal{S}$ and $\mathcal{M}=\mathcal{S} \ominus \mathcal{N}$. Then, again by Remark 2.12,

$$
\left\|P_{Q, \mathcal{S}}\right\|=\left\|P_{Q}, \mathcal{M}\right\|=\left(1-\left\|(1-Q) P_{\mathcal{M}}\right\|^{2}\right)^{-1 / 2}=\left(1-c(N(Q), \mathcal{S})^{2}\right)^{-1 / 2} .
$$


3. Formulas for $P_{A, S}$. This section is devoted to presenting several explicit formulas for $P_{A, \mathcal{S}}$ in terms of the orthogonal projectors onto $\mathcal{S}, \mathcal{W}=A(\mathcal{S})^{\perp} \ominus(\mathcal{S} \cap N(A))$ and $\mathcal{W}^{\perp}$. Afriat [1], Greville [32] and Pták [49] have proven this type of formulas, the first two in finite dimensional settings. Some of these formulas seem to have been known by V. E. Ljance [43]. Consider $A \in L(\mathcal{H})^{+}$and $\mathcal{S}$ a closed subspace of $\mathcal{H}$ such that $(A, \mathcal{S})$ is compatible. Denote $\mathcal{N}=\mathcal{S} \cap A(\mathcal{S})^{\perp}=\mathcal{S} \cap N(A)$ and $\mathcal{W}=A(\mathcal{S})^{\perp} \ominus \mathcal{N}$. Then,

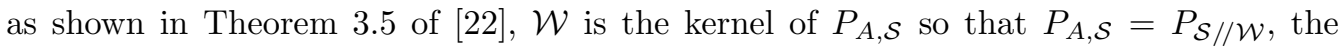
oblique projector onto $\mathcal{S}$, along $\mathcal{W}$. Afriat [1] and Greville [32] exhibited formulas for an oblique projector $Q$ in terms of the orthogonal projectors onto $R(Q)$ and $N(Q)$, by using the Moore-Penrose pseudoinverse. However, in order to use the same method in our infinite dimensional setting we need to know that the operator whose Moore-Penrose pseudoinverse is considered has closed range [23]. This justifies the need of a proof for the first part of the next result. The rest of the proof follows without change Greville's arguments.

\section{LEMMA 3.1.}

1. $(A, \mathcal{S})$ is compatible if and only if $P_{\mathcal{W}} P_{\mathcal{S}}$ has closed range.

2. If the pair $(A, \mathcal{S})$ is compatible then

(a) $P_{A, \mathcal{S}}=\left(P_{\mathcal{W} \perp} P_{\mathcal{S}}\right)^{\dagger}$.

(b) $P_{A, \mathcal{S}}=\left(I-P_{\mathcal{S}} P_{\mathcal{W}}\right)^{-1} P_{\mathcal{S}}\left(I-P_{\mathcal{S}} P_{\mathcal{W}}\right)$.

(c) $P_{A, \mathcal{S}}=\left(I-P_{\mathcal{W}} P_{\mathcal{S}}\right)^{-1}\left(I-P_{\mathcal{W}}\right)=P_{\mathcal{S}}\left(P_{\mathcal{S}}+P_{\mathcal{W}}-P_{\mathcal{W}} P_{\mathcal{S}}\right)^{-1}$.

Proof. If $(A, \mathcal{S})$ is compatible then $\mathcal{H}=\mathcal{S} \oplus \mathcal{W}$ by the remarks above. Observe first that $R\left(P_{\mathcal{W}} P_{\mathcal{S}}\right)=\mathcal{W}^{\perp}$ : for this, it suffices to show the inclusion $\mathcal{W}^{\perp} \subseteq R\left(P_{\mathcal{W}^{\perp}} P_{\mathcal{S}}\right)$, because the converse is evident. If $\xi \in \mathcal{W}^{\perp}$, then $\xi$ decomposes as $\xi=\sigma+\omega, \sigma \in \mathcal{S}$ and $\omega \in \mathcal{W}$, so that $\xi=P_{\mathcal{W} \perp} x=P_{\mathcal{W} \perp} \sigma \in P_{\mathcal{W} \perp} \mathcal{S}=R\left(P_{\mathcal{W} \perp} P_{\mathcal{S}}\right)$.

Conversely, if $P_{\mathcal{W} \perp} P_{\mathcal{S}}$ has closed range then $\left(P_{\mathcal{W} \perp} P_{\mathcal{S}}\right)^{\dagger}$ is a bounded linear operator. Greville's arguments for matrices [32] can be used almost without changes to prove that $\left(P_{\mathcal{W}} P_{\mathcal{S}}\right)^{\dagger}$ is an idempotent with range $\mathcal{S}$ and kernel $\mathcal{W}$. Then $\mathcal{H}=\mathcal{S} \oplus \mathcal{W}=\mathcal{S}+A(\mathcal{S})^{\perp}$ and $(A, \mathcal{S})$ is compatible. The formulas of part 2 follow from the fact that $P_{A, \mathcal{S}}=P_{\mathcal{S} / / \mathcal{W}}$, using Theorem 2.2 .

Corollary 3.2. If the pair $(A, \mathcal{S})$ is compatible and $\mathcal{N}=\{0\}$ then $P_{A, \mathcal{S}}=\left(P_{\overline{A(\mathcal{S})}} P_{\mathcal{S}}\right)^{\dagger}=$ $\left(I-P_{\mathcal{S}} P_{A(\mathcal{S})^{\perp}}\right)^{-1} P_{\mathcal{S}}\left(I-P_{\mathcal{S}} P_{A(\mathcal{S})^{\perp}}\right)=\left(I-P_{A(S) \perp} P_{\mathcal{S}}\right)^{-1}\left(I-P_{A(\mathcal{S})^{\perp}}\right)$.

The shorted operator of $A$ to $\mathcal{S}$ is $A / \mathcal{S}=\sup \left\{X \in L(\mathcal{H})^{\dagger}: X \leq A\right.$ and $\left.R(X) \subseteq \mathcal{S}\right\}$. In [48], Pekarev proved

$$
A / \mathcal{S}^{\perp}=A^{1 / 2} P_{\mathcal{M}^{\perp}} A^{1 / 2},
$$

where $\mathcal{M}=\overline{A^{1 / 2}(\mathcal{S})}$. Let us show a formula for $P_{A, \mathcal{S}}$ in the spirit of Pekarev's. The relationship between the projectors in $\mathcal{P}(A, \mathcal{S})$ and $A / \mathcal{S}^{\perp}$ is given by the formula $A / \mathcal{S}^{\perp}=$ $A E$, which holds for every projector $E$ such that $I-E \in \mathcal{P}(A, \mathcal{S})$ (see [19]). In particular $A / \mathcal{S}^{\perp}=A\left(I-P_{A, \mathcal{S}}\right)$ and, if $A$ were invertible, we can compute

$$
P_{A, \mathcal{S}}=A^{-1}\left(A-A \mathcal{S}^{\perp}\right)=A^{-1 / 2} P_{\mathcal{M}} A^{1 / 2} .
$$

In order to get a generalization of this formula, we consider firstly the injective case: 
Proposition 3.3. Let $A \in L(\mathcal{H})^{+}$injective such that $(A, \mathcal{S})$ is compatible. Then

$$
P_{A, \mathcal{S}}=A^{-1 / 2} P_{\mathcal{M}} A^{1 / 2}
$$

where $\mathcal{M}=\overline{A^{1 / 2}(\mathcal{S})}$.

Proof. Observe that in this case $\mathcal{P}(A, \mathcal{S})=\left\{P_{A, \mathcal{S}}\right\}$ because $\mathcal{S} \cap N(A)=\{0\}$. Define $Q=$ $A^{-1 / 2} P_{\mathcal{M}} A^{1 / 2}$. Then $Q$ is well defined because $A^{-1 / 2}: R\left(A^{1 / 2}\right) \rightarrow \mathcal{H}$ and $R\left(P_{\mathcal{M}} A^{1 / 2}\right) \subseteq$ $R\left(A^{1 / 2}\right)$, by Theorem 2.14. It is easy to see that $Q^{2}=Q$ and that $N(Q)=A(\mathcal{S})^{\perp}$ : in fact, $\xi \in N(Q)$ if and only if $P_{\mathcal{M}} A^{1 / 2} \xi=0$, i.e., $A^{1 / 2} \xi \in A^{1 / 2}(\mathcal{S})^{\perp}$, or, what is the same, $\xi \in A^{-1 / 2}\left(A^{-1 / 2}\left(\mathcal{S}^{\perp}\right)\right)=A^{-1}\left(\mathcal{S}^{\perp}\right)$. On the other hand, by the definition of $Q$, $R(Q) \subseteq A^{-1 / 2}(\mathcal{M})=A^{-1 / 2}\left(\overline{A^{1 / 2}(\mathcal{S})}\right)=A^{-1 / 2}\left(\overline{A^{1 / 2}(\mathcal{S})} \cap R\left(A^{1 / 2}\right)\right)=A^{-1 / 2}\left(A^{1 / 2}(\mathcal{S})\right)=$ $\mathcal{S}$ because, by Theorem $2.13, A^{1 / 2}(\mathcal{S})$ is closed in $R\left(A^{1 / 2}\right)$; this proves that $R(Q) \subseteq \mathcal{S}$. Conversely, if $\sigma \in \mathcal{S}$, then $Q \sigma=A^{-1 / 2} P_{\mathcal{M}} A^{1 / 2} \sigma=\sigma$. Then $R(Q)=\mathcal{S}$ and $Q=P_{A, \mathcal{S}}$.

We generalize this formula to any (not necessarily injective) $A \in L(\mathcal{H})^{+}$. For $B \in$ $L(\mathcal{H})^{+}$denote

$$
B^{\sharp}=\left(\left.B\right|_{\overline{R(B)}}\right)^{-1}: R(B) \rightarrow \overline{R(B)} \subseteq \mathcal{H} .
$$

Observe that $B^{\sharp}$ is a linear, not necessarily bounded operator. If $R(B)$ is closed, then $B^{\sharp} P_{R(B)}=B^{\dagger}$.

Proposition 3.4. Consider $A \in L(\mathcal{H})^{+}$such that $(A, \mathcal{S})$ is compatible. Set $\mathcal{M}=\overline{A^{1 / 2}(\mathcal{S})}$.

1. If $\mathcal{S} \subseteq \overline{R(A)}$ then $P_{A, \mathcal{S}}=\left(A^{1 / 2}\right)^{\sharp} P_{\mathcal{M}} A^{1 / 2}$.

2. If $\mathcal{S} \cap N(A)=\{0\}$ then $P_{A, \mathcal{S}}=\left(P_{\overline{R(A)}} P_{\mathcal{S}}\right)^{\dagger} P_{A, P_{\overline{R(A)}}}(\mathcal{S})=\left(P_{\overline{R(A)}} P_{\mathcal{S}}\right)^{\dagger}\left(A^{1 / 2}\right)^{\sharp} P_{\mathcal{M}} A^{1 / 2}$.

Proof. Observe that $\mathcal{P}(A, \mathcal{S})=\left\{P_{A, \mathcal{S}}\right\}$ because $\mathcal{S} \cap N(A)=\{0\}$ in both cases.

1. If $\mathcal{S} \subseteq \overline{R(A)}$ and $Q=\left(A^{1 / 2}\right)^{\sharp} P_{\mathcal{M}} A^{1 / 2}$ then $Q$ is well defined because $P_{\mathcal{M}}\left(R\left(A^{1 / 2}\right)\right)$ $\subseteq R\left(A^{1 / 2}\right)$, by Proposition 2.14. On one hand $P_{\mathcal{M}}\left(R\left(A^{1 / 2}\right)\right) \subseteq \mathcal{M} \cap R\left(A^{1 / 2}\right)=A^{1 / 2}(\mathcal{S})$, because, by Remark 2.13, $A^{1 / 2}(\mathcal{S})$ is closed in $R\left(A^{1 / 2}\right)$ thus, $R(Q) \subseteq\left(A^{1 / 2}\right)^{\sharp} A^{1 / 2}(\mathcal{S})=\mathcal{S}$. On the other hand, $Q \sigma=\sigma$, for all $\sigma \in \mathcal{S}$, because $\mathcal{S} \subseteq \overline{R(A)}$. Then $R(Q)=\mathcal{S}$. It is easy to see that $N(Q)=A^{-1}\left(\mathcal{S}^{\perp}\right)$; thus, $Q=P_{A, \mathcal{S}}$.

2. If $\mathcal{S} \cap N(A)=\{0\}$ then the subspace $\mathcal{S}^{\prime}=P_{\overline{R(A)}}(\mathcal{S})$ is closed because $(A, \mathcal{S})$ is compatible, $\mathcal{S}^{\prime} \subseteq \overline{R(A)}$ and $\left(A, \mathcal{S}^{\prime}\right)$ is compatible (see Proposition 2.13). Also $\overline{A^{1 / 2}\left(\mathcal{S}^{\prime}\right)}=$ $\overline{A^{1 / 2}(\mathcal{S})}=\mathcal{M}$, so that $P_{A, \mathcal{S}^{\prime}}=\left(A^{1 / 2}\right)^{\sharp} P_{\mathcal{M}} A^{1 / 2}$. Now, $R\left(P_{\overline{R(A)}} P_{\mathcal{S}}\right)=\mathcal{S}^{\prime}$ is closed and $N\left(P_{\overline{R(A)}} P_{\mathcal{S}}\right)=\mathcal{S}^{\perp}$ : the proof is straightforward.

In the general case the set $\mathcal{P}(A, \mathcal{S})$ can be parametrized by means of the set of complements $\mathcal{L}$ of $\mathcal{N}=\mathcal{S} \cap N(A)$ in $\mathcal{S}$. More precisely:

Proposition 3.5. Let $Q \in \mathcal{Q}$ and consider $A \in L(\mathcal{H})^{+}$such that $(A, \mathcal{S})$ is compatible. Let $\mathcal{N}=\mathcal{S} \cap N(A)$. Then $Q \in \mathcal{P}(A, \mathcal{S})$ if and only if there exists a (unique) closed subspace $\mathcal{L} \subseteq \mathcal{S}$ such that $\mathcal{S}=\mathcal{N} \oplus \mathcal{L}, \mathcal{L}+N(Q)$ is closed, $\mathcal{S}+N(Q)=\mathcal{H}$ and

$$
Q=P_{A, \mathcal{L}}+P_{\mathcal{N} / /(\mathcal{L}+N(Q))} \text {. }
$$

Proof. $\Leftarrow)$ Observe that $\mathcal{N}+\mathcal{L}+N(Q)=\mathcal{S}+N(Q)=\mathcal{H}$ and $\mathcal{L}+N(Q)$ is closed so that $Q^{\prime}=P_{\mathcal{N} / /(\mathcal{L}+N(Q))}$ is a well defined (oblique) projector. If $Q=P_{A, \mathcal{L}}+Q^{\prime}$ then it is easy to see that $Q \in \mathcal{P}(A, \mathcal{S})$. 
$\Rightarrow$ Consider $Q \in \mathcal{P}(A, \mathcal{S})$ and let $W=P_{\mathcal{N}} Q$; then $R(W)=\mathcal{N}$. From $Q P_{\mathcal{N}}=P_{\mathcal{N}}$ we get that $W^{2}=W$. Let $T=Q-W ; T$ is $A$-selfadjoint because $Q$ and $W$ are both $A$-selfadjoint; equality $T^{2}=T$ follows from $Q W=W=W Q$. Therefore $Q=T+W$, with $T^{2}=T$ and $W^{2}=W$. Let $\mathcal{L}=\mathcal{S} \cap N(W)$. It follows easily that $T=P_{A, \mathcal{L}}, \mathcal{S}=\mathcal{L}+\mathcal{N}$ and $N(W)=\mathcal{L}+N(Q)$.

Let $C \in L(\mathcal{H})$ such that $R(C)=\mathcal{S}$ is closed, and $A \in L(\mathcal{H})^{+}$with closed range. Formula (3) suggests the natural generalization, which is widely used in the finite dimensional case:

$$
P_{A, \mathcal{S}} \stackrel{?}{=} C\left(C^{*} A C\right)^{\dagger} C^{*} A .
$$

In general, the formula is false for many reasons. For instance, $\left(C^{*} A C\right)^{\dagger}$ is unbounded if $R\left(C^{*} A C\right)$ is not closed; or $C\left(C^{*} A C\right)^{\dagger} C^{*} A$ may have range strictly contained in $\mathcal{S}$. However, the wide range of applications of the right side of formula (4) makes it desirable to establish its exact relationship with $P_{A, \mathcal{S}}$. In fact, projectors like $C\left(C^{*} A C\right)^{\dagger} C^{*} A$ appear explicitly in papers on scaled projections [53], [46], [34], [31], [60], [14], linear least squares problems [28], [29], linear feasibility [28], [29], [17], signal processing [36], [10], [58] and so on.

A first observation is that one needs to verify if $R\left(C^{*} A C\right)$ is closed. An interesting fact, which generalizes item 2 of Remark 2.12, is that $R\left(C^{*} A C\right)$ is closed if and only if $(A, \mathcal{S})$ is compatible. Indeed, note that $R\left(C^{*} A C\right)$ is closed if and only if $R\left(A^{1 / 2} C C^{*} A^{1 / 2}\right)$ is closed. Since $R(C)=\mathcal{S}$ is closed, there exist $a, b>0$ such that $a P \leq C C^{*} \leq b P$, so that

$$
a A^{1 / 2} P A^{1 / 2} \leq A^{1 / 2} C C^{*} A^{1 / 2} \leq b A^{1 / 2} P A^{1 / 2} .
$$

This implies, by the Douglas theorem, the identity

$$
R\left(\left(A^{1 / 2} C C^{*} A^{1 / 2}\right)^{1 / 2}\right)=R\left(A^{1 / 2} P\right)=A^{1 / 2}(\mathcal{S}),
$$

which is closed if and only if $(A, \mathcal{S})$ is compatible, by Remark 2.12 .

Suppose now that $(A, \mathcal{S})$ is compatible. If $\mathcal{N}=N(A) \cap \mathcal{S}$, we shall see that

$$
P_{A, \mathcal{S}}=P_{\mathcal{N}}+C\left(C^{*} A C\right)^{\dagger} C^{*} A,
$$

showing that formula (4) holds if and only if $N(A) \cap \mathcal{S}=\{0\}$.

Define $Q=C\left(C^{*} A C\right)^{\dagger} C^{*} A$. It is clear that $Q^{2}=Q, R(Q) \subseteq R(C)=\mathcal{S}$ and $A Q=$ $Q^{*} A$. Therefore, $Q$ is an $A$-selfadjoint projector onto a subspace of $\mathcal{S}$. Also, since $C$ and $\left(C^{*} A C\right)^{\dagger}$ are injective on $R\left(C^{*}\right)$,

$$
N(Q)=N\left(C^{*} A\right)=A^{-1}\left(N\left(C^{*}\right)\right)=A^{-1}\left(\mathcal{S}^{\perp}\right) .
$$

The next step is to show that $R(Q)=\mathcal{S} \ominus \mathcal{N}$. Note that

$$
R\left(C^{*} A\right)=C^{*}(R(A))=C^{*}\left(R\left(A^{1 / 2}\right)\right)=R\left(C^{*} A^{1 / 2}\right) .
$$

Hence $R\left(C^{*} A\right)=R\left(\left(C^{*} A C\right)^{\dagger} C^{*} A^{1 / 2}\right)$ and $R(Q)=R\left(C C^{*} A^{1 / 2}\right)=R\left(C C^{*} A\right)$. But

$$
N\left(A C C^{*}\right)=N\left(C C^{*}\right) \perp\left(R\left(C C^{*}\right) \cap N(A)\right)=\mathcal{S}^{\perp} \perp \mathcal{N},
$$

so that $R(Q)=N\left(A C C^{*}\right)^{\perp}=\mathcal{S} \ominus \mathcal{N}$, as claimed. This fact clearly shows that $Q \in$ $\mathcal{P}(A, \mathcal{S} \ominus \mathcal{N})=\left\{P_{A, \mathcal{S} \ominus \mathcal{N}}\right\}$ (since $\left.(\mathcal{S} \ominus \mathcal{N})^{\perp_{A}}=\mathcal{S}^{\perp_{A}}\right)$ and also proves formula (5). 
It is shown in [6] that for every projector $Q$ onto a closed subspace $\mathcal{S}$, there exists an invertible positive $A \in L(\mathcal{H})$ such that $Q=P_{A, \mathcal{S}}$. This can be rewritten as follows:

Proposition 3.6. Let $\mathcal{S} \in \mathcal{H}$ be a closed subspace and $C \in L(\mathcal{H})$ with $R(C)=\mathcal{S}$. Let $A \in L(\mathcal{H})^{+}$with closed range. Then

1. $(A, \mathcal{S})$ is compatible if and only if $R\left(C^{*} A C\right)$ is closed.

2. If $N(A) \cap \mathcal{S}=\mathcal{N}$, then

$$
P_{A, \mathcal{S}}=P_{\mathcal{N}}+C\left(C^{*} A C\right)^{\dagger} C^{*} A .
$$

3. For every $Q \in L(\mathcal{H})$ such that $Q^{2}=Q$ and $R(Q)=\mathcal{S}$, there exists an invertible positive $A \in L(\mathcal{H})$ such that

$$
Q=C\left(C^{*} A C\right)^{-1} C^{*} A
$$

Final comments and open problems. The structure of the set $\mp \mathcal{S}=\left\{A \in L(\mathcal{H})^{+}\right.$: $(A, \mathcal{S})$ is compatible $\}$ is not completely known. We have observed that $G L(\mathcal{H})^{+}$is contained in $\mp \mathcal{S}$. Of course, if $\mathcal{S}$ is finite-dimensional, then $\mp \mathcal{S}=L(\mathcal{H})^{+}$.

The extension of compatibility questions to Hermitian operators instead of positive operators is a much more difficult problem. The reader can find in [35], [19] and [44] some results in this direction.

Compatibility is related to some problems arising from wavelet and frame theory. The paper [7] deals with some problems in this area.

A difficult and very useful problem consists in determining conditions which ensure the convergence of sequences like $\left\{P_{A_{n}, \mathcal{S}}\right\}$ and $\left\{P_{A, \mathcal{S}_{n}}\right\}$. A sample of this type of results can be found in [21].

Given $Q \in \mathcal{Q}^{\mathcal{S}}$, it is known that $\chi_{Q}=\left\{A \in L(\mathcal{H})^{+}: Q \in \mathcal{P}(A, \mathcal{S})\right\}$ is not empty and the set $\chi_{Q} \cap G L(\mathcal{H})^{+}$is characterized [6]. However, in general, the structure of $\chi_{Q}$ is unknown and it would be interesting to have optimality criteria for choosing $A \in \chi_{Q}$.

\section{References}

[1] S. N. Afriat, Orthogonal and oblique projectors and the characteristics of pairs of vector spaces, Proc. Cambridge Philos. Soc. 53 (1957), 800-816.

[2] A. Aldroubi, Oblique projections in atomic spaces, Proc. Amer. Math. Soc. 124 (1996), 2051-2060.

[3] A. Aldroubi, Oblique and hierarchical multiwavelet bases, Appl. Comput. Harmon. Anal. 4 (1997), 231-263.

[4] W. N. Anderson and G. E. Trapp, Shorted operators II, SIAM J. Appl. Math. 28 (1975), $60-71$.

[5] T. Ando, De Branges spaces and analytic operator functions, Research Institute of Applied Electricity, Hokkaido University, Sapporo, Japan, (1990).

[6] E. Andruchow, G. Corach and D. Stojanoff, Geometry of oblique projections, Studia Math. 137 (1999), 61-79.

[7] J. Antezana, G. Corach, M. A. Ruiz and D. Stojanoff, Weighted projectors and Riesz frames, Linear Algebra Appl., in press. 
[8] M. Atteia, Généralisation de la définition et des propriétés des "spline fonctions", C. R. Acad. Sci. Paris 260 (1965), 3550-3553.

[9] J. K. Baksalary and R. Kala, Two relations between oblique and $\Lambda$-orthogonal projections, Linear Algebra Appl. 24 (1979), 99-103.

[10] R. T. Behrens and L. L. Scharf, Signal processing applications of oblique projection operators, IEEE Trans. Signal Process. 42 (1994), 1413-1424.

[11] J. Benedetto, Frames, sampling, and seizure prediction, in: Advances in Wavelets (Hong Kong, 1997), Springer, Singapore, 1999, 1-25.

[12] A. Ben-Israel and T. N. E. Greville, Generalized Inverses: Theory and Applications, Robert E. Krieger Publishing Co., Inc., Huntington, N.Y., 1980.

[13] T. Blu and M. Unser, Quantitative Fourier analysis of approximation techniques. I. Interpolators and projections, IEEE Transactions on Signal Processing 47 (1999), 2783-2795.

[14] E. Bobrovnikova and S. A. Vavasis, A norm bound for projectors with complex weights, Linear Algebra Appl. 307 (2000), 69-75.

[15] L. de Branges and J. Rovnyak, Square Summable Power Series, Holt, Rinehart and Winston, New York, 1966.

[16] D. Buckholtz, Hilbert space idempotents and involutions, Proc. Amer. Math. Soc. 128 (2000), 1415-1418.

[17] Y. Censor and T. Elfving, Block-iterative algorithms with diagonally scaled oblique projections for the linear feasibility problem. SIAM J. Matrix Anal. Appl. 24 (2002), 40-58.

[18] K. Y. Chung, Subspaces and graphs, Proc. Amer. Math. Soc. 119 (1993), 141-146.

[19] G. Corach, A. Maestripieri and D. Stojanoff, Schur complements and oblique projections, Acta Sci. Math. (Szeged) 67 (2001), 439-459.

[20] G. Corach, A. Maestripieri and D. Stojanoff, Generalized Schur complements and oblique projections, Linear Algebra Appl. 341 (2002), 259-272.

[21] G. Corach, A. Maestripieri and D. Stojanoff, Oblique projections and abstract splines, Journal of Approximation Theory 117 (2002), 189-206.

[22] C. Davis, Separation of two linear subspaces, Acta Sci. Math. (Szeged) 19 (1958), 172-187.

[23] C. A. Desoer and B. H. Whalen, A note on pseudoinverses, Journal of Society for Industrial and Applied Mathematics 11 (1963), 442-447.

[24] F. Deutsch, The angle between subspaces in Hilbert space, in: pproximation theory, wavelets and applications, S. P. Singh, (ed.), Kluwer, 1995, 107-130.

[25] J. Dieudonné, Quasi-hermitian operators, in: Proc. Internat. Symp. Linear Spaces, Jerusalem (1961), 115-122.

[26] R. G. Douglas, On majorization, factorization and range inclusion of operators in Hilbert space, Proc. Amer. Math. Soc. 17 (1966), 413-416.

[27] P. A. Fillmore and J. P. Williams, On operator ranges, Advances in Math. 7 (1971), $254-281$.

[28] A. Forsgren, On linear least-squares problems with diagonally dominant weight matrices. SIAM J. Matrix Anal. Appl. 17 (1996), 763-788.

[29] A. Forsgren and G. Sporre, On weighted linear least-squares problems related to interior methods for convex quadratic programming, SIAM J. Matrix Anal. Appl. 23 (2001), 42-56.

[30] W. Gerisch, Idempotents, their Hermitian components, and subspaces in position $p$ of a Hilbert space, Math. Nachr. 115 (1984), 283-303.

[31] C. C. Gonzaga and H. J. Lara, A note on properties of condition numbers, Linear Algebra Appl. 261 (1997), 269-273. 
[32] T. N. E. Greville, Solutions of the matrix equations $X A X=X$ and relations between oblique and orthogonal projectors, SIAM J. Appl. Math. 26, 4 (1974), 828-832.

[33] P. R. Halmos, Two subspaces, Trans. Amer. Math. Soc. 144 (1969), 381-389.

[34] M. Hanke and M. Neumann, The geometry of the set of scaled projections, Linear Algebra Appl. 190 (1993), 137-148.

[35] S. Hassi and K. Nordström, On projections in a space with an indefinite metric, Linear Algebra Appl. 208/209 (1994), 401-417.

[36] S. Kayalar and H. L. Weinert, Oblique projections: formulas, algorithms and error bounds, Math. Control Signal Systems 2 (1989), 33-45.

[37] T. Kato, Perturbation Theory for Linear Operators, Reprint of the 1980 edition, Classics in Mathematics, Springer-Verlag, Berlin, 1995.

[38] N. Kerzman and E. M. Stein, The Szegő kernel in terms of Cauchy-Fantappiè kernels, Duke Math. J. 45 (1978), 197-224.

[39] N. Kerzman and E. M. Stein, The Cauchy kernel, the Szegö kernel, and the Riemann mapping function, Math. Ann. 236 (1978), 85-93.

[40] M. G. Krein, The theory of self-adjoint extensions of semibounded Hermitian operators and its applications, Mat. Sb. (N.S.) 20 (62) (1947), 431-495.

[41] P. D. Lax, Symmetrizable linear transformations, Comm. Pure Appl. Math. 7 (1954), 633-647.

[42] C. Lee, M. Eden and M. Unser, High quality image resizing using oblique projection operators, IEEE Trans. Image Proces. 7 (1998), 679-692.

[43] V. E. Ljance, Certain properties of idempotent operators, Teoret. Prikl. Mat. Vyp. 1 (1958), 16-22 (in Russian).

[44] S. K. Mitra and C. R. Rao, Projections under seminorms and generalized Moore Penrose inverses, Linear Algebra Appl. 9 (1974), 155-167.

[45] V. J. Mizel and M. M. Rao, Nonsymmetric projections in Hilbert space, Pacific J. Math. 12 (1962), 343-357.

[46] D. P. O'Leary, On bounds for scaled projections and pseudoinverses, Linear Algebra Appl. 132 (1990), 115-117.

[47] Z. Pasternak-Winiarski, On the dependence of the orthogonal projector on deformations of the scalar product, Studia Math. 128 (1998), 1-17.

[48] E. L. Pekarev, Shorts of operators and some extremal problems, Acta Sci. Math. (Szeged) 56 (1992), 147-163.

[49] V. Pták, Extremal operators and oblique projections, Časopis pro pestování Matematiky 110 (1985), 343-350.

[50] Ju. L. Smuljan, An operator Hellinger integral, Mat. Sb. (N.S.) 49 (91) (1959), 381-430 (in Russian).

[51] J. Steinberg, A class of integral transforms which are projections, Integral Equations Operator Theory 34 (1999), 65-90.

[52] J. Steinberg, Oblique projections in Hilbert spaces, Integral Equations Operator Theory 38 (2000), 81-119.

[53] G. W. Stewart, On scaled projections and pseudoinverses, Linear Algebra Appl. 112 (1989), 189-193.

[54] Y. Takane and H. Yanai, On oblique projectors, in: Linear Algebra and Statistics (Istanbul, 1997), Linear Algebra Appl. 289 (1999), 297-310.

[55] W. S. Tang, Oblique projections, biorthogonal Riesz bases and multiwavelets in Hilbert spaces, Proc. Amer. Math. Soc. 128 (2000), 463-473. 
[56] W. S. Tang, Oblique multiwavelets in Hilbert spaces, Proc. Amer. Math. Soc. 128 (2000), 2017-2031.

[57] M. Unser, Quasi-orthogonality and quasi-projections, Applied Comp. Harmonic Anal. 3 (1996), 201-214.

[58] P. Vandaele and M. Moonen, Two deterministic blind channel estimation algorithms based on oblique projectors, Signal Process. 80 (2000), 481-495.

[59] M. J. Vrhel, C. Lee and M. Unser, Rapid computation of the continuous wavelet transform by oblique projectors, IEEE Trans. Signal Process. 45 (1997), 891-900.

[60] M. S. Wei, Upper bound and stability of scaled pseudoinverses, Numer. Math. 72 (1995), 285-293.

[61] H. K. Wimmer, Canonical angles of unitary spaces and perturbations of direct complements, Linear Algebra Appl. 287 (1999), 373-379.

[62] H. K. Wimmer, Lipschitz continuity of oblique projectors, Proc. Amer. Math. Soc. 128 (1999), 873-876.

[63] X. Yu and L. Tong, Joint channel and symbol estimation by oblique projectors, IEEE Transactions on Image Processing 8 (1999), 527-536. 